\title{
REPRESENTATION OF AN ABSTRACT MEASURE USING BOREL-ISOMORPHISM TYPES
}

\author{
R. M. SHORTT
}

(Communicated by R. Daniel Mauldin)

\begin{abstract}
For $X \subseteq R$, the mapping $t$ which assigns to each Borel subset of $X$ its isomorphism type is an abstract measure. Given a monoid-valued measure $m$, we ask when there is an $X \subseteq R$ such that $t$ and $m$ are isomorphic as measures.
\end{abstract}

Given a set $X \subseteq R$, we consider the mapping $B \rightarrow t(B)$ associating to each relative Borel subset $B$ of $X$ its Borel-isomorphism type $t(B)$. This mapping may be considered as an abstract measure taking values in a cardinal algebra, or more generally, in a partially ordered monoid. Using techniques developed by Chuaqui [2], the author has noted [4] that in certain special circumstances, this measure may be represented by an ordinary probability measure $m$ :

$$
t\left(B_{1}\right) \leq t\left(B_{2}\right) \text { iff } m B_{1} \leq m B_{2} .
$$

In this paper, the inverse idea is explored: given a (not necessarily real-valued) measure $m$, can one find a set $X$ so that $(*)$ holds? This generalizes earlier work [5], which considered only real-valued measures. We employ the continuum hypothesis $(\mathrm{CH})$.

Each subset $X$ of $\mathbf{R}$ inherits a relative Borel structure ( $\sigma$-field)

$$
\mathscr{B}(X)=\{B \cap X: B \subseteq \mathbf{R}, B \text { Borel }\},
$$

the elements of which we call measurable subsets of $X$. Two such subsets $X_{1}$ and $X_{2}$ are Borel-isomorphic if there is a one-one correspondence $f: X_{1} \rightarrow X_{2}$ with $A \in \mathscr{B}\left(X_{1}\right)$ if and only if $f(A) \in \mathscr{B}\left(X_{2}\right)$. Each $X \subseteq R$ determines an isomorphism type

$$
t(X)=\left\{\begin{array}{l}
\{Y \subseteq R: Y \text { and } X \text { isomorphic }\} ; \quad X \text { uncountable } \\
0 ; \quad X \text { countable }
\end{array}\right.
$$

Put $S=\{t(X): X \subseteq R\}$. We introduce algebraic and order structures on $S$ as follows. Given $t_{1}$ and $t_{2}$ in $S$, let $X_{1} \subseteq(0,1)$ and $X_{2} \subseteq(1,2)$ be sets with

Received by the editors December 12, 1987 and, in revised form, August 1, 1988.

1980 Mathematics Subject Classification (1985 Revision). Primary 28A05; Secondary 04A15, 28B10.

Key words and phrases. Borel isomorphism, cardinal algebra, monoid, measure. 
$t\left(X_{1}\right)=t_{1}$ and $t\left(X_{2}\right)=t_{2}$. Put $t_{1}+t_{2}=t\left(X_{1} \cup X_{2}\right)$. A similar device serves to define $t_{1}+t_{2}+\cdots$ for a sequence of types in $S$. Define $t_{1} \leq t_{2}$ in case $t_{2}=t+t_{1}$ for some $t \in S$.

It was noted by Tarski [6; pp. 234-235, passim] that under the above operations, $S$ constitutes a cardinal algebra, and for each $X \subseteq R$, the subset $\{t(Y): Y \in \mathscr{B}(X)\}$ is a generalized cardinal algebra. For information about cardinal algebras, see [6].

A partial isomorphism of $\mathbf{R}$ is a Borel-isomorphism between sets in $\mathscr{B}(\mathbf{R})$. Given such a partial isomorphism $f$ and any $A \subseteq \mathbf{R}$, we define

$$
\begin{gathered}
f^{-1}(A)=\{x \in \operatorname{dom}(f): f(x) \in A\} \\
f(A)=\{f(x): x \in \operatorname{dom}(f) \cap A\} .
\end{gathered}
$$

Note that $A$ need not be a subset of the domain or range of $f$. Let $H$ be a collection of partial isomorphisms of $\mathbf{R}$. A set $A \subseteq \mathbf{R}$ is complete for $H$ if each $h$ in $H$ maps $\operatorname{dom}(h) \cap A$ onto range $(h) \cap A$.

We note that a Borel-isomorphism between arbitrary subsets of $\mathbf{R}$ extends to a Borel-isomorphism between Borel subsets of $\mathbf{R}$ (i.e., a partial isomorphism of $\mathbf{R})$. See [3; p. 436]. Also, any two uncountable members of $\mathscr{B}(\mathbf{R})$ are Borel-isomorphic. See [3; p. 450].

By a commutative divisibility monoid $[1 ;$ p. 320] is meant a set $\mathscr{M}$ together with a binary operation + such that:

(1) Commutative and associative laws hold for + .

(2) There is in $\mathscr{M}$ an identity element 0 for + .

(3) Define a relation $\leq$ on $\mathscr{M}$ by saying that $a \leq b$ iff $b=a+c$ for some $c$ in $\mathscr{M}$. This relation should be a partial order on $\mathscr{M}$.

We write $a_{1}+a_{2}+\cdots=\sup \left(a_{1}+\cdots+a_{n}\right)$ whenever such a supremum exists in $\mathscr{M}$. We note that every cardinal algebra as well as the nonnegative cone of any Abelian partially ordered group forms a commutative divisibility monoid.

Let $(X, \mathscr{B})$ be a measurable space and suppose that $m: \mathscr{B} \rightarrow \mathscr{M}$ is a function taking values in a commutative divisibility monoid. Say that $m$ is a measure if

$$
m\left(A_{1} \cup A_{2} \cup \cdots\right)=m A_{1}+m A_{2}+\cdots
$$

for every pairwise disjoint sequence of sets $A_{n}$ in $\mathscr{B}$; the supremum on the right-hand side is assumed to exist. Ordinary Lebesgue measure (with $\mathscr{M}=$ $[0, \infty])$ is one example of such; another is any measure taking values in the positive cone of an ordered vector space. The most important example for this paper, however, is the following: Let $X$ be an arbitrary subset of $\mathbf{R}$ and define $m: \mathscr{B}(\mathbf{R}) \rightarrow S$ by $m B=t(B \cap X)$. Then $m$ is a measure taking values in the cardinal algebra $\mathscr{M}=S$.

Let $m: \mathscr{B}(\mathbf{R}) \rightarrow \mathscr{M}$ be a measure taking values in $\mathscr{M}$. Say that $m$ is continuous if $m\{p\}=0$ for each $p \in \mathbf{R}$. Call $m$ completely homogeneous if whenever $B_{1}$ and $B_{2}$ are Borel subsets of $\mathbf{R}$ with $m B_{1}=m B_{2}>0$, then there 
are Borel subsets $B_{1}^{\prime}$ and $B_{2}^{\prime}$ of $\mathbb{R}$ such that $B_{1}^{\prime} \subseteq B_{1}, B_{2}^{\prime} \subseteq B_{2}, m\left(B_{1}-B_{1}^{\prime}\right)=$ $m\left(B_{2}-B_{2}^{\prime}\right)=0$, and such that there is a Borel-isomorphism $h$ of $B_{1}^{\prime}$ onto $B_{2}^{\prime}$ with $m A=m h(A)$ for each measurable $A \subseteq B_{1}^{\prime}$. Finally, consider the condition

(M) Whenever $B_{1}$ and $B_{2}$ are Borel subsets of $\mathbf{R}$ with $m B_{1} \leq$ $m B_{2}$, there is some Borel $C \subseteq B_{2}$ with $m B_{1}=m C$.

This condition seems to have arisen in some early work of Maharam.

Consider once more the measure $m$ on $\mathscr{B}(\mathbf{R})$ defined by $m B=$ $t(B \cap X)$. Clearly, $m$ is continuous, completely homogeneous and satisfies condition (M). The main result below shows that (under $\mathrm{CH}$ ) every measure on $\mathscr{B}(\mathbf{R})$ satisfying these conditions is isomorphic to one of the form $m B=$ $t(B \cap X)$ above.

Theorem (CH). Let $m$ be a continuous, completely homogeneous measure on $\mathscr{B}(\mathbf{R})$ taking values in a commutative divisibility monoid $\mathscr{M}$. Then there is a set $X \subseteq \mathbf{R}$ such that

$$
t\left(B_{1} \cap X\right)=t\left(B_{2} \cap X\right) \text { iff } m B_{1}=m B_{2}
$$

whenever $B_{1}$ and $B_{2}$ are Borel subsets of $\mathbf{R}$.

If $m$ satisfies condition $(\mathrm{M})$, then we also have

$$
t\left(B_{1} \cap X\right) \leq t\left(B_{2} \cap X\right) \text { iff } m B_{1} \leq m B_{2} .
$$

Proof. Let $H$ be the set of all partial isomorphisms $h$ on $\mathbf{R}$ such that $m h(A)=$ $m A$ for each measurable set $A \subseteq \operatorname{dom}(h)$. List the elements of $H$ in transfinite series as $h_{0} h_{1} \cdots h_{\alpha} \cdots \alpha<\omega_{1}$, insisting that $h_{0}$ be the identity map on $\mathbf{R}$. Next, list as $N_{0} N_{1} \cdots N_{\alpha} \cdots\left(\alpha<\omega_{1}\right)$ and $P_{0} P_{1} \cdots P_{\alpha} \cdots\left(\alpha<\omega_{1}\right)$ those Borel subsets $N$ and $P$ of $\mathbb{R}$ such that $m N=0$ and $m P>0$. Finally, consider the collection of all partial isomorphisms $k$ such that $m(\operatorname{dom}(k)) \$ m(\operatorname{range}(k))$. List such isomorphisms as $k_{0} k_{1} \cdots k_{\alpha} \cdots \alpha<\omega_{1}$.

For each countable ordinal $\alpha$ and $x \in \mathbf{R}$, let $\mathscr{O}_{\alpha}(x)$ be the smallest subset of $R$ containing $x$ and complete for the collection $\left\{h_{0} h_{1} \cdots h_{\alpha}\right\}$. Fix $\alpha$ and define $F$ to be the set of all partial isomorphisms of the form

$$
f=h_{\beta_{1}}^{n_{1}} \circ h_{\beta_{2}}^{n_{2}} \circ \cdots \circ h_{\beta_{k}}^{n_{k}},
$$

where $n_{1} \cdots n_{k}$ are integers and $\beta_{1} \cdots \beta_{k}$ are ordinals not greater than $\alpha$. Then $F$ is countable and may be listed as $f_{1} f_{2} \cdots$. It is not hard to verify that

$$
\mathscr{O}_{n}(x)=\left\{f_{n}(x): x \in \operatorname{dom}\left(f_{n}\right), n \geq 1\right\} \text {. }
$$

Given $A \subseteq \mathbf{R}$, define $\mathscr{O}_{\alpha}(A)=\bigcup\left\{\mathscr{O}_{\alpha}(x): x \in A\right\}$. We see that $\mathscr{O}_{c}(A)$ is the smallest subset of $R$ containing $A$ and complete for $\left\{h_{0} \cdots h_{\alpha}\right\}$. From this follows

Claim 1. If $A$ is countable [resp. $m A=0$ ], then $\mathscr{O}_{a}(A)$ is countable [resp. $m \mathscr{O}_{i}(A)=0$ ]. We also make 
Claim 2. Suppose that $k$ is a partial isomorphism on $\mathbf{R}$ with $m(\operatorname{dom}(k)) \not$ $m($ range $(k))$. Then $m\left\{x \in \operatorname{dom}(k): k(x) \notin \mathscr{O}_{\alpha}(x)\right\}>0$.

Proof of claim. Define sets $A_{1} A_{2} \cdots$ by setting

$$
\begin{aligned}
& A_{1}=\left\{x \in \operatorname{dom}\left(f_{1}\right) \cap \operatorname{dom}(k): k(x)=f_{1}(x)\right\}, \\
& A_{n}=\left\{x \in \operatorname{dom}\left(f_{n}\right) \cap \operatorname{dom}(k): k(x)=f_{n}(x)\right\}-\bigcup_{1}^{n-1} A_{k} .
\end{aligned}
$$

Also put $A_{0}=\left\{x \in \operatorname{dom}(k): k(x) \notin \mathscr{O}_{\alpha}(x)\right\}$. Noting that $h_{0}$ (and therefore one of the $f_{n}$ 's) is the identity map on $\mathbf{R}$, we see that $\mathbf{R}=\bigcup \operatorname{dom}\left(f_{n}\right)$ and

$$
\operatorname{dom}(k)=A_{0} \cup A_{1} \cup A_{2} \cup \cdots
$$

as a disjoint union. Thus $A_{0}$ is measurable. If now $m A_{0}=0$, then

$$
\begin{aligned}
m(\operatorname{range}(k)) & \geq m k\left(A_{1}\right)+m k\left(A_{2}\right)+\cdots \\
& =m f_{1}\left(A_{1}\right)+m f_{2}\left(A_{2}\right)+\cdots \\
& =m A_{0}+m A_{1}+m A_{2}+\cdots \\
& =m(\operatorname{dom}(k))
\end{aligned}
$$

is a contradiction. The claim is proved.

Now choose points $x_{\alpha}$ and $y_{\alpha}$ for $\alpha<\omega_{1}$ so that

$$
\begin{aligned}
x_{\alpha} \in \operatorname{dom}\left(k_{\alpha}\right) & -\mathscr{O}_{\alpha}\left(N_{0} \cup \cdots \cup N_{\alpha}\right) \\
& -k_{\alpha}^{-1}\left(\mathscr{O}_{\alpha}\left\{x_{\beta}, y_{\beta}: \beta<\alpha\right\}\right) \\
& -k_{\alpha}^{-1}\left(\mathscr{O}_{\alpha}\left(x_{\alpha}\right)\right) \\
& -\mathscr{O}_{\alpha}\left\{k_{\beta}\left(x_{\beta}\right): \beta<\alpha\right\}
\end{aligned}
$$

and

$$
\begin{aligned}
y_{\alpha} \in P_{\alpha} & -\mathscr{O}_{\alpha}\left(N_{0} \cup \cdots \cup N_{\alpha}\right) \\
& -\mathscr{O}_{\alpha}\left\{k_{\beta}\left(x_{\beta}\right): \beta \leq \alpha\right\} .
\end{aligned}
$$

Claims 1 and 2 guarantee the possibility of such choices. Define $X=$ $\bigcup\left\{\mathscr{O}_{\alpha}\left(x_{\alpha}, y_{\alpha}\right): \alpha<\omega_{1}\right\}$.

Claim 3. For each countable $\alpha$, we have $X \cap N_{\alpha} \subseteq \bigcup\left\{\mathscr{O}_{\beta}\left(x_{\beta}, y_{\beta}\right): \beta<\alpha\right\}$. Thus, $X \cap N_{\alpha}$ is countable.

Proof of claim. Suppose that $u \in N_{\alpha} \cap \mathscr{O}_{\beta}\left(x_{\beta}, y_{\beta}\right)$ for some $\beta \geq \alpha$. Then

$$
u \in \mathscr{O}_{\beta}\left(x_{\beta}, y_{\beta}\right) \cap \mathscr{O}_{\beta}\left(N_{0} \cup \cdots \cup N_{\alpha} \cup \cdots \cup N_{\beta}\right)
$$

implies that either $x_{\beta}$ or $y_{\beta}$ is a member of $\mathscr{O}_{\beta}\left(N_{0} \cup \cdots \cup N_{\beta}\right)$, a contradiction which proves the claim.

Claim 4. For each $\alpha$, the intersection $X \cap P_{\alpha}$ is uncountable.

Proof of claim. Suppose not. Then because $m\left(P_{\alpha}-\left(X \cap P_{\alpha}\right)\right)+m\left(X \cap P_{\alpha}\right)=$ $m P_{\alpha}>0$, we have $P_{\alpha}-\left(X \cap P_{\alpha}\right)=P_{\beta}$ for some $\beta$. But $X \cap P_{\beta}$ is nonvoid (it contains $\left.y_{\beta}\right)$, a contradiction. 
Claim 5. Suppose that $B_{1}$ and $B_{2}$ are Borel subsets of $R$ with $m B_{1}=m B_{2}$. Then $t\left(B_{1} \cap X\right)=t\left(B_{2} \cap X\right)$.

Proof of claim. If $m B_{1}=m B_{2}=0$, then Claim 3 implies that $B_{1} \cap X$ and $B_{2} \cap X$ are countable, so that $t\left(B_{1} \cap X\right)=t\left(B_{2} \cap X\right)=0$. Now suppose that $m B_{1}=m B_{2}>0$. Complete homogeneity for $m$ implies that there is some $\alpha$ with $\operatorname{dom}\left(h_{\alpha}\right) \subseteq B_{1}$, range $\left(h_{\alpha}\right) \subseteq B_{2}$, and

$$
m\left(B_{1}-\operatorname{dom}\left(h_{\alpha}\right)\right)=m\left(B_{2}-\operatorname{range}\left(h_{\alpha}\right)\right)=0 .
$$

The set $X_{0}=\bigcup\left\{\mathscr{O}_{\beta}\left(x_{\beta}, y_{\beta}\right): \beta \geq \alpha\right\}$ is complete for $h_{\alpha}$, so that

$$
\begin{aligned}
h_{\alpha}\left(X_{0} \cap B_{1} \cap X\right) & =h_{\alpha}\left(X_{0} \cap B_{1}\right) \\
& =X_{0} \cap B_{2} \\
& =X_{0} \cap\left(B_{2} \cap X\right) .
\end{aligned}
$$

Now $\left(B_{1} \cap X\right)-X_{0}$ and $\left(B_{2} \cap X\right)-X_{0}$, being contained in $X-X_{0}$, are countable. Thus $t\left(B_{1} \cap X\right)=t\left(B_{2} \cap X\right)$.

Claim 6. Suppose that $B_{1}$ and $B_{2}$ are Borel subsets of $\mathbf{R}$ with $t\left(B_{1} \cap X\right)=$ $t\left(B_{2} \cap X\right)$. Then $m B_{1}=m B_{2}$.

Proof of claim. We prove that $m B_{1} \leq m B_{2}$ and appeal to symmetry. Proceeding absurdi causa, suppose that $\left.m B_{1}\right\rfloor m B_{2}$. Then $m B_{1}>0$, so that (by Claim 4) $B_{1} \cap X$ is uncountable. Thus also $B_{2} \cap X$ is uncountable. Let $f$ be a Borelisomorphism of $B_{1} \cap X$ onto $B_{2} \cap X$. Then $f$ is the restriction to $B_{1} \cap X$ of some Borel-isomorphism $k$ such that

(i) $C_{1}=\operatorname{dom}(k)$ is a Borel subset of $B_{1}$,

(ii) $C_{2}=\operatorname{range}(k)$ is a Borel subset of $B_{2}$.

Then $m B_{i}=m C_{i}+m\left(B_{i}-C_{i}\right)=m C_{i}$ for $i=1,2$, using Claim 4. Since $m C_{1} \$ m C_{2}$, we have that $k=k_{\alpha}$ for some $\alpha<\omega_{1}$. But then $x_{\alpha} \in C_{1} \cap X=$ $B_{1} \cap X$. However, $k_{\alpha}\left(x_{\alpha}\right) \notin \mathscr{O}_{\beta}\left(x_{\beta}, y_{\beta}\right)$ for any $\beta<\alpha$. Also $k_{\alpha}\left(x_{\alpha}\right) \notin$ $\mathscr{O}_{\alpha}\left(x_{\alpha}\right)$. Suppose that $k_{\alpha}\left(x_{\alpha}\right) \in \mathscr{O}_{\beta}\left(x_{\beta}\right)$ for $\beta>\alpha$. Then $x_{\beta} \in \mathscr{O}_{\beta}\left(k_{\alpha}\left(x_{\alpha}\right)\right)$ is a contradiction. Finally, suppose that $k_{\alpha}\left(x_{\alpha}\right) \in \mathscr{O}_{\beta}\left(y_{\beta}\right)$ for $\beta>\alpha$. Then $y_{\beta} \in \mathscr{O}_{\beta}\left(k_{\alpha}\left(x_{\alpha}\right)\right)$, another contradiction.

We have shown that $k_{\alpha}\left(x_{\alpha}\right) \notin X$, which contradiction establishes the claim.

Claims 5 and 6 prove the first part of the theorem.

Suppose now that $m$ satisfies condition (M). If $t\left(B_{1} \cap X\right) \leq t\left(B_{2} \cap X\right)$, then either $B_{1} \cap X$ is countable, in which case $m B_{1}=0$, or else $B_{1} \cap X$ is Borel-isomorphic with $C \cap X$, where $C$ is some measurable subset of $B_{2}$. So $m B_{1}=m C \leq m B_{2}$. On the other hand, suppose that $m B_{1} \leq m B_{2}$. Condition (M) implies that there is some Borel set $C \subseteq B_{2}$ with $m C=m B_{1}$. Then

$$
t\left(B_{1} \cap X\right)=t(C \cap X) \leq t\left(B_{2} \cap X\right),
$$

as desired. Q.E.D.

Corollary $(\mathrm{CH})$. Let $m$ be a continuous, completely homogeneous measure on $\mathscr{B}(\mathbf{R})$ taking values in a commutative divisibility monoid $\mathscr{M}$. Then the range of $m$ is a generalized cardinal algebra (in the sense of Tarski [6]). 
Indication. Choose $X$ as in the theorem. As noted previously, $\{t(B \cap X)$ : $B \subseteq \mathbb{R}$ Borel $\}$ is a generalized cardinal algebra. The mapping $t(B \cap X) \rightarrow m B$ is well defined and determines an isomorphism onto $\operatorname{range}(m)$.

The following result generalizes Proposition 6.6 in [5].

Corollary $(\mathrm{CH})$. Given $I=[0,1]$ and $n=1,2, \ldots, \infty$, consider the cube $I^{n}$ under coordinate-wise addition and partial order. There is a set $X \subset \mathbf{R}$ with $\{t(Y): Y \in \mathscr{B}(X)\}$ and $I^{n}$ isomorphic (with respect to both addition and order). Indication. Let $\mu_{1} \mu_{2} \cdots \mu_{n}$ be the restrictions of Lebesgue measure to the respective intervals $(0,1),(1,2), \ldots,(n-1, n)$. (For $n=\omega$, use an infinite sequence.) Put $m B=\left(\mu_{1} B, \mu_{2} B, \ldots, \mu_{n} B\right)$ and apply the theorem.

Note. If $m(B)=\lambda(B \cap(0,1))$, where $\lambda$ is Lebesgue measure, then any set $X \subset \mathbf{R}$ as in the theorem is a Sierpinski subset of $(0,1)$. It follows that the assumption of $\mathrm{CH}$ cannot be dropped from the theorem.

\section{ACKNOWLEDGMENT}

The author wishes to express thanks to Ralph Kopperman for some stimulating conversations on this topic. The author also thanks the referee for a critical observation.

\section{REFERENCES}

1. G. Birkhoff, Lattice theory, 3rd ed., Amer. Math. Soc. Colloq. Publ. 25, 1967.

2. R. Chuaqui, Cardinal algebras and measures invariant under equivalence relations, Trans. Amer. Math. Soc. 142 (1969), 61-79.

3. K. Kuratowski, Topology, Vol. I, Academic Press, New York, 1966.

4. R. M. Shortt, Representation of Borel-isomorphism by a probability measure, Proc. Amer. Math. Soc. 104 (1988), 284-286.

5. —_ Measurable spaces with c.c.c. Dissertationes Mathematicae (to appear).

6. A. Tarski, Cardinal algebras, Oxford University Press, New York, 1949.

Department of Mathematics, Wesleyan University, Middletown, Connecticut 06457 